\title{
A KSAO Based Solvers' Competency Model in Crowdsourcing Contests
}

\author{
Qingliang Meng1,*, Guicheng $\mathrm{Yu}^{2}$ \\ ${ }^{1}$ School of Economics and Management, Jiangsu University of Science and Technology, Zhenjiang, China \\ ${ }^{2}$ School of Economics and Management, Jiangsu University of Science and Technology, Zhenjiang, China \\ *Corresponding author. Email: mengzhi007@163.com
}

\begin{abstract}
As typical crowdsourcing innovation, crowdsourcing contests have the characteristics such as clear task objectives, intense timelines, and voluntary participation of solvers. It is critical for improving crowdsourcing contests' efficiency to evaluate solvers' competency scientifically and identify appropriate solvers rapidly. The competency model was constructed from four dimensions of Knowledge, Skill, Ability, and Others. This framework will benefit not only the solvers but also the seekers and the crowdsourcing platform.
\end{abstract}

Keywords: Crowdsourcing contests, KSAO model, Competency.

\section{INTRODUCTION}

Crowdsourcing innovation refers to the transfer of innovation tasks traditionally performed by internal employees to external network users in a free and voluntary manner to reduce innovation risks and improve innovation performance. As typical crowdsourcing innovation, crowdsourcing contests have become a valuable way for enterprises (seekers) to obtain innovative solutions at low cost and participants (solvers) to improve themselves and attract widespread attention. In this model, seekers publish time-limited innovation tasks, filter solutions, and finally pay the prize; meanwhile, solvers choose tasks, submit solutions, and eventually win the prize.

Crowdsourcing contests have become a two-subject innovation problem-solving cycle, which has attracted widespread attention. For example, 99designs.com generates an idea every two seconds on average, and there are now 97.34 million ideas generated in 192 countries. The total number of tasks of epwk.com has reached 9.75 million, and solvers 22.33 million. The employer center of zbj.com covers 10 million seekers and aggregates 14 million solvers.

For the surge in the number of users and tasks, the platforms usually display tasks based on a single factor such as the posting time or the prize amount, and provide an input box for solvers searching tasks personally. But there are still hundreds of tasks displayed on the pages, and solvers mostly choose tasks that appear on the first few pages of the task list to participate in [1], which not only wastes solvers' time but may also miss more suitable tasks. Further, there may be a risk that no one or only a few people participate in some tasks, making the tasks hard to complete and making seekers experience poor directly. Ultimately, solvers and seekers churn.

Based on the above, it is an effective strategy to improve both participants' satisfaction, speed up the task cycle, and improve the platform efficiency to evaluate solvers' competency accurately and matching the two participants efficiently in the crowdsourcing contests.

We propose a framework to recommend tasks that a solver is most likely to choose. Considering the factors such as contests' characteristics and solvers' emotions, we construct the competency model from four dimensions: Knowledge, Skill, Ability, and Others. Then the recommendation lists for solvers are generated by a hybrid recommendation method. Finally, through the experiment on data from epwk.com, the framework's feasibility and effectiveness are verified.

\section{LITERATURE REVIEW}

\subsection{Crowdsourcing}

Crowdsourcing innovation creates high value for enterprises and individuals and thus has been studied in various aspects. Based on CiteSpace, Zhu et al. (2019) concluded that the hotspots in crowdsourcing innovation 
focus on three aspects: connotation of open innovation and crowdsourcing innovation, the performance of crowdsourcing innovation, and the crowdsourcing platforms [2].

Existing research on the recommendation system in crowdsourcing, a field of the crowdsourcing platforms mentioned above, has focused mainly on cooperative crowdsourcing, in which a seeker distributes the task into several small parts, and multiple solvers will work together to complete the task. Relevant studies under this mode are sufficient whether it is recommended for solvers [3], seekers [4], even to recommend partners [5]. However, there is relatively less research under competitive crowdsourcing [6], in which a seeker releases a task on the crowdsourcing contest platform, and the solver, as an individual or an organization, submits the solutions before the deadline, the solver whose solution is chosen is the winner and wins the prize in the end.

The recommendation system in crowdsourcing could effectively improve the efficiency of a solver finding the right task and a seeker getting a satisfactory solution. Meanwhile, it could indirectly increase the platform's income, promote the development of the platform, and form a benign closed loop, which is worth studying.

\subsection{Solver Model}

The recommendation framework needs to predict solvers' future behavior and interest according to their history. Therefore, the solver model is critical and directly affects the quality of the system.

For this reason, some scholars have carried out relevant studies from the identification of crucial knowledge sources in crowdsourcing innovation, the influencing factors of the solvers' innovation performance, and the solvers' features, etc. Meng et al. (2017) come up with a crucial knowledge source identification system to identify the solver that matches the seeker from the bilateral perspectives of seekers and solvers [7]. Javadi et al. (2014) proved that the solvers' participation and winning history, including participation frequency, participation status, winning frequency, winning status, final performance, etc., have a significant impact on their innovation performance [8]. Zhang et al. (2020) identify crucial solvers in knowledge-intensive crowdsourcing from demographic features, interest, ability, reputation, and the possibility of participation [9]. Zhang et al. (2019) also integrated typical features of solvers from the bottom up to identify different solvers in knowledge-intensive crowdsourcing [10].

Some scholars choose mature models such as the Competency model and Campbell model to evaluate users. Lv et al. (2013) use the KSA model to evaluate solvers from their knowledge, skill, and service capacity, providing theoretical support for seekers to select appropriate solvers [11]. Zhong et al. (2017) use text mining to estimate solvers' interests, combined with the KSAO model to evaluate solvers' competency as a new solver model, which provided a foundation for the recommendation later [12]. Mo et al. (2018) supplemented the Campbell model with theories from learning, motivation, and tournaments to describe solvers' performance from six dimensions: intrinsic ability, expertise, general experience, specialization, diversity of experience, and complexity of experience [13]. Models help considering the key factors affecting solvers' behavior comprehensively; meanwhile, it is critical to supplement and optimize the model based on the actual environment.

Given the KSAO model has the advantages with flexibility, multi-dimensional, comprehensive evaluation, ease of implementation, etc., we choose this model and combine it with the seekers' willingness to build the solvers' competency model of crowdsourcing contests.

\section{COMPETENCY FRAMEWORK}

\subsection{Necessity}

Crowdsourcing contests' process involves seekers posting the problem on the platform and hosting the prize, solvers choosing the preferred task and submitting the solution before the deadline, seekers screen and evaluate the solutions received. Finally, the platform promotes cooperation between seekers and selected solvers.

As seen from the above process, crowdsourcing contests are characterized by clear task objectives, intense timelines, and voluntary participation of solvers. Therefore, an effective management mechanism should be designed to achieve rapid matching between solvers and seekers, improve competition efficiency, and reduce innovation risks. And the effective evaluation of solvers' competency is the basis of it.

It is beneficial to identify the solver that matches the task quickly. In crowdsourcing contests, solver sources and have diverse knowledge, the solutions they submitted will be kinds of types, and hard to distinguish and evaluate the quality of the solutions quickly. Screening and evaluating all submitted solutions without evaluating the solver first will lead to inefficiencies and higher costs. By effectively assessing the solver's competency, identifying the key solver, and forcing seekers to focus on the solution matched with them, operational efficiency and innovation performance can be greatly improved.

It helps solvers to select tasks efficiently and improve capabilities accordingly. The task's intense timeliness requires solvers to select and quickly 
complete the task before the deadline efficiently. Thus, it requires solvers to have a comprehensive understanding of their ability, estimate the possibility of their ability to win in the competition, and then quickly locate the matching crowdsourcing task. Simultaneously, combined with their competency evaluation results, solvers can improve their ability in crowdsourcing contests.

To sum up, it is necessary to evaluate the competency of solvers.

\subsection{KSAO Model-based Competency of Solvers}

The competency model is a set of indicators to identify and distinguish high-performing individuals and low-performing individuals in a group. Mirabile (1997) constructed the KSAO model to evaluate competency from four dimensions [14]: Knowledge, Skill, Ability, and Others. Knowledge refers to a series of experiences necessary to complete a task, which

Table 1. Competency of solvers in crowdsourcing contests affects the completion quality of the work directly; Skill refers to the demonstration of some particular skills, especially the effective output of the knowledge you have; Ability refers to competencies that are endowed by nature which could also be formed through acquired learning and training, such as dialectical thinking and learning ability, etc.; Others refer to other features required to complete a task efficiently, such as attitude, personality, habits, etc. We propose the KSAO modelbased competency of solvers in crowdsourcing contests, as shown in Table 1.

Knowledge describes the professional knowledge that a solver required to complete a particular task, and it is used to evaluate if the solver suits the task. Seekers often consider the expertise solvers reported and refer to the tasks that the solver has participated historically and the solutions submitted. Therefore, Bid Type and Report Type can be used to describe the solvers' knowledge.

Bid Type (BT) refers to the tasks' type that solver bid:

\begin{tabular}{|l|l|l|}
\hline Dimensions & Indicators & Descriptions \\
\hline \multirow{2}{*}{ Knowledge } & Bid Type & the types of tasks that solver bid \\
\cline { 2 - 3 } & Report Type & the types of tasks that the solver reports he can do \\
\hline \multirow{2}{*}{ Skill } & Bid Rate & the bid rate of each type of tasks that the solver bid \\
\cline { 2 - 3 } & Success Rate & the success rate of each type of tasks that the solver bid \\
\hline \multirow{2}{*}{ Ability } & Service Capacity & the speed, quality, and attitude of service \\
\cline { 2 - 3 } & Competitiveness & store grade, total success rate, the number of shop followers \\
\hline \multirow{2}{*}{ Others } & Deposit & the amount of deposit solver paid \\
\cline { 2 - 3 } & Certification & whether real name authentication and can be contacted
\end{tabular}

$B T=\left(B T_{1}, B T_{2}, \ldots B T_{i}, \ldots B T_{n}\right), B T_{i} \in\{0,1\}, i=1,2, \ldots, n$, where $\mathrm{n}$ is equal to the number of task types.

Report Type (RT) refers to the types of tasks that the solver reports he can do, then

$R T=\left(R T_{1}, R T_{2}, \ldots R T_{i}, \ldots R T_{n}\right), R T_{i} \in\{0,1\}, i=1,2, \ldots, n$.

Skill describes solvers' proficiency in using a particular technology or tool, which affects the quality of the solutions submitted by solvers directly. Crowdsourcing contests as a time-limited competition, making it more likely that solvers, who can produce quality solutions efficiently, will win. However, neither in the crowdsourcing process nor in the platform's database, there are no indicators that directly measure solvers' technical proficiency. Considering the actual situation, we proposed two arrows, Bid Rate and Success Rate, to describe the technical ability.

Bid Rate (BR) refers to the bid rate of each type of task that the solver bid, then

$$
B R=\left(B R_{1}, B R_{2}, \ldots B R_{i}, \ldots B R_{n}\right), i=1,2, \ldots, n .
$$

Success Rate $(S R)$ refers to the success rate of each type of task that the solver bid, then

$$
S R=\left(S R_{1}, S R_{2}, \ldots S R_{i}, \ldots S R_{n}\right), i=1,2, \ldots, n .
$$

Ability describes the quality required to complete a particular task, which is the point to barge the solver to the forefront. In addition to considering whether the solver can complete the task and whether the task can be perfectly completed, the solver's work attitude, problemsolving speed, and whether he is competitive are also essential that seekers will think over for efficient collaboration. Therefore, we describe solvers' ability to provide quality service and better solutions with Service Capacity (SC) and Competitiveness $(C P)$ respectively.

$$
\begin{aligned}
& S C=(S S, S Q, S A), \\
& C P=(S G, T R, S F) .
\end{aligned}
$$

Others describe the additional features that solvers required to accomplish a task. When choosing solvers, seekers will consider some other related factors to ensure the task will come to a successful conclusion, such as whether the transaction is safe enough (whether the task can be completed, whether there is after-sale service, whether the solutions are original, etc.) and whether the identity information of solvers is accurate, etc. In practice, the crowdsourcing platform usually sets up some indicators to quantify the relevant information of solvers. So, we choose Deposit (DP) and Certification $(C F)$ to indicate the problems above.

To sum up, the competency set of solver $P$ can be expressed as

$\mathrm{Com}_{P}=\left(B T_{P}, R T_{P}, B R_{P}, S R_{P}, S C_{P}, C P_{P}, D P_{P}, C F_{P}\right)$. 


\section{CONCLUSION}

Our research makes several contributions, we propose a KSAO based solvers' competency model in crowdsourcing contests, which takes both solvers' competency and seekers' favor into account. In which, Bid Type and Report Type describe the Knowledge dimension, Bid Rate and Success Rate describe the Skill dimension, Service Capacity and Competitiveness describe the Ability dimension, and Deposit and Certification describe the Others dimension.

Our work has an excellent guiding significance for realizing the matching between solvers and tasks. With our model, a recommendation in crowdsourcing contests could improve both seekers' and solvers' satisfaction and the platform's benefit.

\section{ACKNOWLEDGMENTS}

The authors thank the editor and the referees for carefully reading the paper. This work was supported by the MOE Layout Foundation of Humanities and Social Sciences 19YJA630055, Postgraduate Research \& Practice Innovation Program of Jiangsu Province KYCX20_3168.

\section{REFERENCES}

[1] L. B. Chilton, J. J. Horton, R. C. Miller et al., Task search in a human computation market, in: Proceedings of the ACM SIGKDD workshop on human computation, ACM, NEW YORK, USA, 2010, $\quad$ pp. $1-9 . \quad$ DOI: https://doi.org/10.1145/1837885.1837889

[2] B. X. Zhu, Z. Q. Ma, Knowledge Maps Analysis of Crowdsourcing Innovation Based on CiteSpace, Science and Technology Management Research, Guangzhou, Guangdong, China, 2019, pp. 1-9. DOI: 10.3969/j.issn.1000-7695.2019.09.001

[3] P. Mavridis, D. Gross-Amblard, Z. Miklós, Using hierarchical skills for optimized task assignment in knowledge-intensive crowdsourcing, in: Proceedings of the 25th International Conference on World Wide Web, ACM, NEW YORK, USA, 2016, pp. 843-853. DOI: https://doi.org/10.1145/2872427.2883070

[4] Z. Liu, L. Chen, Worker recommendation for crowdsourced q\&a services: A triple-factor aware approach, in: Proceedings of the VLDB Endowment, ACM, NEW YORK, USA, 2017, pp. 380-392.

DOI: https://doi.org/10.14778/3157794.3157805

[5] H. Rahman, S. B. Roy, S. Thirumuruganathan et al., Optimized group formation for solving collaborative tasks, The VLDB Journal,
SPRINGER, NEW YORK, USA, 2019, pp. 1-23. DOI: https://doi.org/10.1007/s00778-018-0516-7

[6] M. R. Karim, Y. Yang, D. Messinger et al., Learn or Earn?-Intelligent Task Recommendation for Competitive Crowdsourced Software Development, in: Hawaii International Conference on System Sciences, 2018, pp. 5604-5613. DOI: https://doi.org/10.24251/HICSS.2018.700

[7] Q. L. Meng, X. X. Guo, Research on Identification of Key Knowledge Source in Crowdsourcing Innovation Based on BP Neural Network, Science of Science and Management of S.\&.T, 2017, pp. 139-148. DOI: CNKI:SUN:KXXG.0.2017-03-013.

[8] H. Javadi Khasraghi, A. Aghaie, Crowdsourcing contests: understanding the effect of competitors' participation history on their performance, Behaviour \& Information Technology, TAYLOR \& FRANCIS LTD, MILTON PARK, OXON, ENGLAND, 2014, pp. 1383-1395. DOI: https://doi.org/10.1080/0144929X.2014.883551

[9] X. F. Zhang, B. G. Gong, Y. Q. Cao et al., Investigating participants' attributes for participant estimation in knowledge-intensive crowdsourcing: a fuzzy DEMATEL based approach, Electronic Commerce Research, 2020, pp. 1-32. DOI: https://doi.org/10.1007/s10660-020-09408-1

[10] X. F. Zhang, B. G. Gong, H. Y. Ni et al., Identifying Participants' Characteristics Influencing Participant Estimation in Knowledge-Intensive Crowdsourcing, in: 2019 8th International Conference on Industrial Technology and Management (ICITM), IEEE, NEW YORK, USA, 2019, pp. 358-363. DOI: https://doi.org/10.1109/ICITM.2019.8710681

[11] Y. J. Lv, P. Z. Zhang, J. F. Liu, Task-oriented Talent Selection in Crowdsourcing, Journal of Systems \& Management, 2013, pp. 60-66. DOI: CNKI:SUN:XTGL.0.2013-01-010.

[12] Q. Y. Zhong, Y. Zhang, C. Li et al., Task recommendation method based on worker's interest and competency for crowdsourcing, Systems Engineering - Theory \& Practice, 2017, pp. 32703280. DOI: CNKI:SUN:XTLL.0.2017-12-023.

[13] J. Mo, S. Sarkar, S. Menon, Know when to run: recommendations in crowdsourcing contests, MIS Quarterly, SOC INFORM MANAGE-MIS RES CENT, MINNEAPOLIS, USA 2018, pp. 919-944. DOI: https://doi.org/10.25300/MISQ/2018/14103

[14] R. J. Mirabile, Everything you wanted to know about competency modeling, Training \& Development, AMER SOC TRAINING DEVELOPMENT, ALEXANDRIA, 1997, pp. 7378. 